\title{
The expression of CD74 and macrophage migration inhibitory factor protein is upregulated in hepatitis B virus-related hepatocellular carcinoma
}

\author{
Mingzhu Lu ${ }^{1 \#}$, Yun $\mathrm{Xu}^{2 \#}$, Guangzhao $\mathrm{He}^{1 \#}$, Qian Liu ${ }^{1}$, Jing Zhu ${ }^{1}$, Changsong Zhang ${ }^{1}$ \\ ${ }^{1}$ Clinical Oncology Laboratory, Changzhou Cancer Hospital, Soochow University, Changzhou 213032, China; ${ }^{2}$ Department of Oncology, Nanyang \\ Center Hospital, Nanyang 473000, China \\ Contributions: (I) Conception and design: C Zhang; (II) Administrative support: C Zhang; (III) Provision of study materials or patients: M Lu, Y Xu; (IV) \\ Collection and assembly of data: Q Liu, G He; (V) Data analysis and interpretation: J Zhu; (VI) Manuscript writing: All authors; (VII) Final approval \\ of manuscript: All authors. \\ "These authors contributed equally to this work. \\ Correspondence to: Dr. Changsong Zhang. No. 68 Honghe Rd. Clinical Oncology Laboratory, Changzhou Cancer Hospital of Soochow University, \\ Changzhou 213032, China. Email: changsong@suda.edu.cn.
}

Background: Pro-inflammatory cytokine production, such as CD74 and macrophage migration inhibitory factor (MIF) could play an important role in liver cancer following chronic hepatitis B virus (HBV) infection.

Methods: The level of CD74 was evaluated by using a capture enzyme-linked immunosorbent assay (ELISA) kit. The level of MIF protein was determined using Human Magnetic Luminex ${ }^{\circledR}$ Assays on 60 HBV-related hepatocellular carcinoma (HCC) patients. The level of HBV copies was determined by using real-time polymerase chain reaction (RT-PCR). Furthermore, $\alpha$-fetoprotein (AFP), alanine aminotransferase (ALT), and aspartate aminotransferase (AST) were determined by the cobas 6000 analyzer.

Results: The mean concentration of CD74 was elevated significantly in the tumor tissues $(10.24 \mathrm{ng} / \mathrm{mg})$ when it was compared with non-tumor tissues $(3.86 \mathrm{ng} / \mathrm{mg}$ ). Meanwhile, the level of MIF was $63.49 \mathrm{ng} / \mathrm{mg}$ in the tumor tissues, and $45.47 \mathrm{ng} / \mathrm{mg}$ in non-tumor samples. We found that elevated CD74 protein expression was associated with an elevated serum AFP level and the AST/ALT ratio; however, an elevated MIF protein level was associated with a lymph node metastasis, serum AFP level and AST/ALT ratio. Only a high level of MIF protein was associated with a short-term survival rate of just months in the HBV-related HCC patients. The elevated expression of MIF protein was associated with $\mathrm{HBV} \geq 10^{5}$ (copies/mL) in liver cancer. The elevated level of MIF protein was associated with the elevated level of CD74 protein in HBVassociated HCC progression.

Conclusions: These results suggest that the elevated level of CD74 and MIF cytokines could play a significant role in HBV-related HCC.

Keywords: Hepatocellular carcinoma (HCC); CD74; macrophage migration inhibitory factor (MIF); $\alpha$-fetoprotein (AFP); hepatitis B virus (HBV)

Submitted Aug 03, 2018. Accepted for publication Nov 15, 2018.

doi: $10.21037 /$ tcr.2018.11.21

View this article at: http://dx.doi.org/10.21037/tcr.2018.11.21 


\section{Introduction}

In China, hepatocellular carcinoma (HCC) is one of most common cancers with complication of chronic hepatitis, especially for the hepatitis B virus (HBV) (1). Increasing evidence suggests that chronic $\mathrm{HBV}$ infection could result in progressive liver disease due to it leading to chronic inflammation of hepatocytes. Cirrhosis could result from the inflammation of liver tissues based on the environment, which can induce a pro-inflammatory cytokine production, to contribute to cancer development $(2,3)$.

As a major histocompatibility complex (MHC) class II chaperone, CD74 is a type of transmembrane protein, which is expressed on the cell surface of antigen-presenting cells. In immune cells, CD74 plays the role of a receptor for the cytokine, macrophage migration inhibitory factor (MIF) (4). MIF is also as a pro-inflammatory cytokine and represents an important link between chronic inflammation and tumorigenesis (5). As a pleiotropic pro-inflammatory cytokine, MIF is expressed by a variety of different cells and tissues, such as T-cells, monocytes/macrophages, endocrine cells, endothelial cells or epithelial cells, participating in inflammatory and immune responses (6). High CD74 expression has been detected in tumor cells and inflammatory cells in bladder cancer, and was significantly associated with older age, high grade, and advanced stages, by which it serves as a prognostic factor (7).

MIF promotes tumor growth and metastasis by increasing the prevalence of the myeloid-derived suppressor cells in the tumor microenvironments (8), and was also found to be overexpressed in several other cancers (9). MIF promoted tumor aggressiveness via the activation of Akt and the inactivation of GSK3 $\beta$ in esophageal squamous cell carcinoma (ESCC) (10). A high level of MIF protein was detected by immunochemistry tests in tumor cells or in tumor-infiltrating lymphocytes, and was demonstrated to be an independent factor for the reduced survival months in ESCC patients (11).

The overexpression of CD74 on chronic lymphocytic cells could act as a transcription regulator involved in immune regulation, cell survival and tumor cell maintenance (12). MIF has been shown to be associated with promoting tumorigenesis by favoring tumor cell proliferation and blocking apoptosis. MIF could bind to CD74 and then activate the phosphatidylinositol-3-kinase/serine/threonine kinase (PI3K/Akt) pathway involved in numerous human malignancies (13). MIF also interacts with surface CD74, inducing its phosphorylation and the recruitment of CD44, leading ultimately to extracellular signal-regulated kinase $1 / 2$ (ERK1/2) phosphorylation (14).

There is also a growing amount of evidence indicating that the MIF-CD74 pathway is involved in carcinogenesis. The expressions of MIF and CD74 were found in $95 \%$ and $98 \%$ of malignant pleural mesothelioma specimens and the elevation of CD74 level was confirmed to be an independent prognostic factor (15). The higher levels of serum MIF was found in breast cancer patients; meanwhile, MIF and CD74 were overexpressed in tumor tissues (16). MIF may promote angiogenesis and metastasis in HCC by increased vascular endothelial growth factor (VEGF) expression, which may be helpful for the treatment of HCC (17).

Although the mechanisms remain unclear, the implied key characteristics of an inflammation are triggered by abnormal microenvironments in the liver which promote HCC's development. To assay the microenvironment changes in HBV-related HCC patients, the expression levels of inflammatory cytokines CD74 and MIF have been quantified in tumors and non-tumor samples. Our aims were to explore the role that the CD74-MIF pathway plays in the pathophysiology of HBV-related liver malignancies.

\section{Methods}

\section{Patients and samples}

Sixty HBV-related HCC patients were included in this study from October 2010 to September 2017. All the patients were recruited from the Changzhou Cancer Hospital of Soochow University in China. HCC patients were diagnosed according to the criteria set by the European Association for the Study of the Liver (EASL). Based on the history of HBV infection, biochemical and serological tests, etc. hematoxylin and eosin (H\&E)-stained slides of tissues were re-examined independently by three experienced pathologists. The patients who had a history of HBV infection for a time frame of $>6$ months were diagnosed with HCC. Blood samples were collected from each patient for routine liver function tests. Meanwhile, tumor and nontumor liver tissues were obtained after surgical operation. The matched non-HCC tissues were obtained from the liver $>3 \mathrm{~cm}$ away from tumors. Patients were from ages 35 to 78 . All of patients gave written informed consent, and approval was given by the Ethics Committee of Changzhou Cancer Hospital of Soochow University (No. 2016006). 


\section{Laboratory assays}

We collected $10 \mathrm{~mL}$ blood sample from each patient during diagnosis. Serum samples were separated and stored at $-80{ }^{\circ} \mathrm{C}$ for the next analysis. Serum alanine aminotransferase (ALT) and aspartate aminotransferase (AST) were routinely detected in our hospitals by the cobas ${ }^{\circledR}$ series 6000 analyzer using commercial kits. Serum HBV DNA was assayed by real-time polymerase chain reaction (RT-PCR) using the COBAS TaqMan Kit (Roche Diagnostics, Branchburg, NJ, USA). Patients with HBV DNA $\left(\geq 10^{5}\right.$ copies/mL) were considered as active chronic carriers.

\section{Quantification of CD74 protein in tissues}

The level of CD74 protein found in the serum was detected by the enzyme-linked immunosorbent assay (ELISA) kit for "RayBio ${ }^{\circledR}$ Human CD 74 ELISA Kit" (Cat. No. ELHCD74, RayBiotech Inc., Peachtree Corners, USA) following the manufacturer's instructions. Tissues samples were lysed using the xTractor Buffer (Code No. 635625, Clontech Laboratories Inc., Mountain View, USA) for proteins. After treatment with lysis, and balance buffers, quantification of the protein concentration was done with the TaKaRa Bradford Protein Assay Kit (Code No. T9310A, Takara Co., Beijing, China).

\section{Quantification of MIF protein in tissues}

The level of MIF protein was determined by using the Human Magnetic Luminex ${ }^{\circledR}$ Assays (LXSAHM, R\&D Systems Inc., Minneapolis, USA) kit according to the manufacturer's instructions. The Magnetic Luminex kit was used with the Luminex $200^{\mathrm{TM}}$ flow-based sorting and detection platforms by the Luminex MAGPIX ${ }^{\circledR}$ CCD Imager. Then, we retrieved the protein level by using a standard curve using a five-parameter logistic (5-PL) curve-fit.

\section{Statistical analysis}

Statistical analysis was performed using SPSS 18.0 (SPSS, Inc., Chicago, USA). Student $t$-test and Chi-square tests were applied to evaluate the differences in the categorical variables. Univariate analysis included the variables like age, smoking, ALT and AST, etc. The correlation analyses between CD74 and MIF were performed with a linear regression model. Methods for survival data analysis were based on the Kaplan-Meier estimates. $\mathrm{P}$ value $<0.05$ and two-sided were considered significant.

\section{Results}

\section{Overexpression of CD74 and MIF in $\mathrm{HBV}$-related $\mathrm{HCC}$}

The CD74 and MIF protein were analyzed and matched with HBV-related HCC and non-tumor samples after surgical operation. The mean concentration of CD74 was elevated significantly in the tumor tissues $(10.24 \mathrm{ng} / \mathrm{mg}$; 95\% CI, 7.81-12.67) when it was compared with nontumor controls (3.86 ng/mg; 95\% CI, 3.09-4.63; $\mathrm{P}<0.0001$, Figure $1 A, B)$. The CD74 ratio means the level of CD74 in tumor/non-tumor; and MIF ratio means the level of CD74 in tumor/non-tumor. We found an elevated level of CD74 in $44(73.33 \%)$ cases (CD74 ratio $\geq 1.0)$, and a decreased level of CD74 in 16 (26.67\%) cases (CD74 ratio <1.0). Meanwhile, the level of MIF was $63.49 \mathrm{ng} / \mathrm{mg}$ (95\% CI, 50.89-76.09) in HBV-related HCC tissues, and $45.47 \mathrm{ng} / \mathrm{mg}$ (95\% CI, 36.06-54.88) in non-tumor samples $(\mathrm{P}=0.0035)$ (Figure 1C,D). We found an elevated level of MIF in 37 (84.09\%) cases (MIF ratio $\geq 1.0$ ), and a decreased level of MIF in $23(15.91 \%)$ cases (MIF ratio <1.0). Thus, the levels of CD74 and MIF protein were significantly higher in the HBV-related HCC patients.

\section{The correlations of CD74 and MIF protein with clinicopathological variables in $\mathrm{HBV}$-related $\mathrm{HCC}$}

The associations between clinicopathological variables and the level of protein status in $60 \mathrm{HBV}$-related HCC patients are summarized in Table 1 . The patients are divided into two groups (CD74-high group vs. CD74-low group; MIF-high group vs. MIF-low group) based on the concentration ratio of tumor/non-tumor (high group, ratio $\geq 1.0$; low group, ratio $<1.0$ ). Analysis of these data sets indicated that CD74 protein expression on $\mathrm{HBV}$-related $\mathrm{HCC}$ was associated with serum $\alpha$-fetoprotein (AFP) level $(\mathrm{P}=0.007)$ and serum AST/ALT ratio $(\mathrm{P}=0.003)$. Subsequently, we also found that the level of MIF protein was associated with a lymph node metastasis $(\mathrm{P}=0.001)$, serum AFP level $(\mathrm{P}=0.003)$ and serum AST/ALT ratio $(\mathrm{P}=0.031)$. The protein expression of $\mathrm{CD} 74$ and MIF was not significantly associated with the other clinicopathological variables in HBV-related HCC patients.

Interestingly, high levels of MIF protein were associated with short-term survival months [18 vs. 26 months, log-rank $\mathrm{P}=0.0026$, hazard ratio (HR): $3.524,95 \% \mathrm{CI}, 1.678-7.398$, 

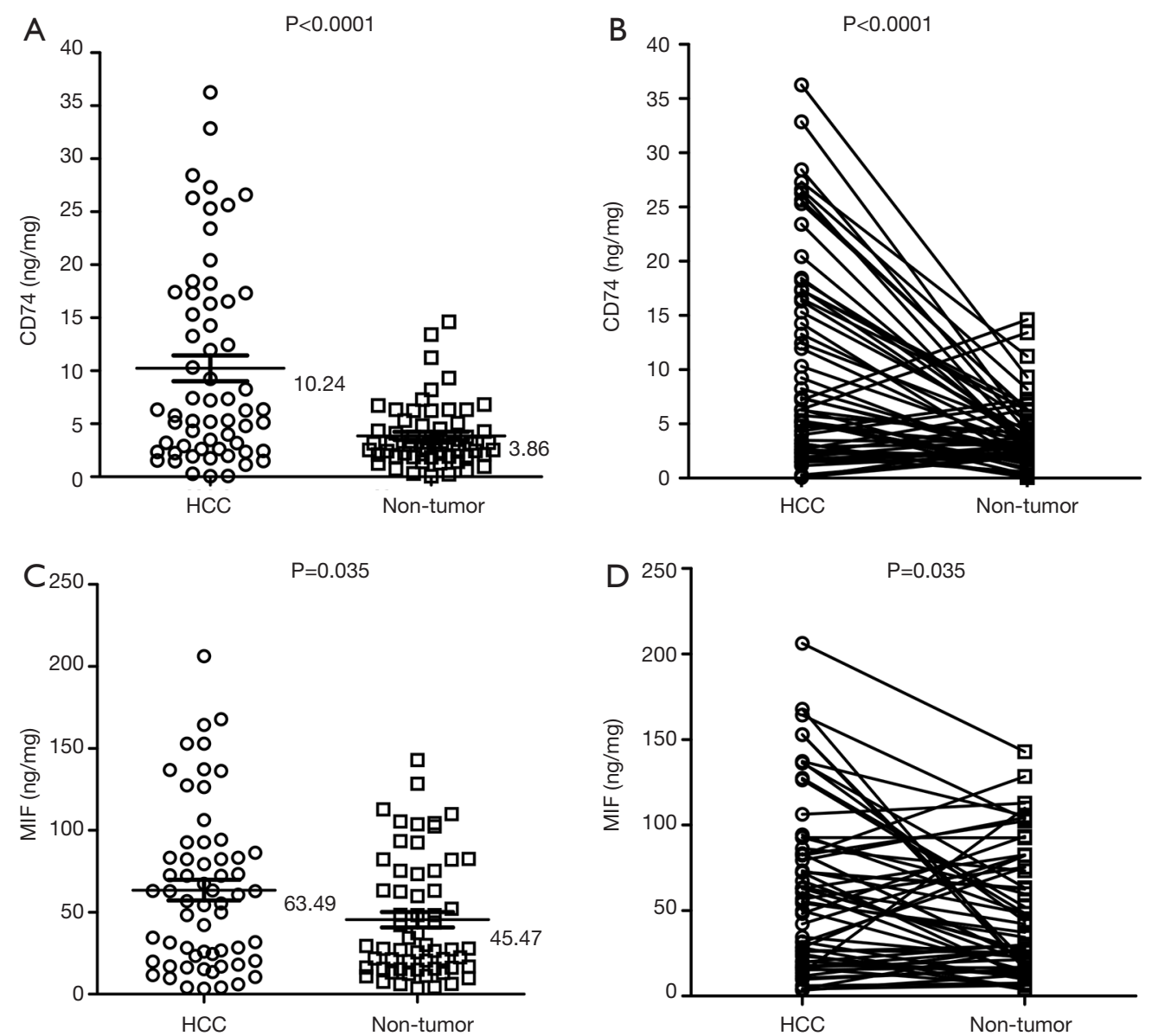

Figure 1 The quantification of CD74 and MIF protein in tissues. (A,B) The quantification of CD74 protein, paired HBV-related HCC and non-tumor tissues; (C,D) the quantification of MIF protein, paired HBV-related HCC and non-tumor tissues. Statistical analysis was done using the paired $t$-test. HBV, hepatitis B virus; MIF, migration inhibitory factor; HCC, hepatocellular carcinoma.

Figure 2A], whereas high levels of CD74 lacked any prognostic significance $(\mathrm{P}=0.7046$, Figure $2 B)$ in $\mathrm{HBV}$-related HCC patients. These results implied a clinical and prognostic relevance of the elevated levels of MIF protein in HCC.

\section{The level of CD74 and MIF protein with serum AFP in $\mathrm{HBV}$-related $\mathrm{HCC}$}

The level of Serum AFP has been used as a tumor marker for the diagnosis of HCC. There were 42 cases $(70.0 \%)$ for the high level of AFP group ( $\mathrm{AFP} \geq 30 \mu \mathrm{g} / \mathrm{L}$ ), and 18 cases $(30.0 \%)$ for the low level of AFP group (AFP $<30 \mu \mathrm{g} / \mathrm{L}$ ). The mean concentration of CD74 was elevated significantly in the AFP-high group (12.97 ng/mg; 95\% CI, 9.93-16.01) when it was compared with the AFP-low group $(3.86 \mathrm{ng} / \mathrm{mg}$;
95\% CI, 1.88-5.83; $\mathrm{P}=0.0003$, Figure $3 A$ ) in the tumor tissue samples. But, the mean concentration of CD74 had no significant difference in the AFP-high group when it was compared with the AFP-low group $(\mathrm{P}=0.1590$, Figure $3 B)$ in the non-tumor tissue samples.

Meanwhile, we found the mean concentration of MIF was elevated significantly in the AFP-high group $(81.97 \mathrm{ng} / \mathrm{mg}$; 95\% CI, 67.28-96.66) when it was compared with the AFPlow group (20.37 ng/mg; 95\% CI, 15.69-25.05; $\mathrm{P}<0.0001$, Figure 3C) in tumor tissue samples. Lastly, the mean concentration of MIF was also elevated significantly in the AFP-high group (52.81 ng/mg; 95\% CI, 41.34-64.28) when compared with the AFP-low group $(28.35 \mathrm{ng} / \mathrm{mg}$; $95 \%$ CI, 13.48-43.21; $\mathrm{P}=0.0158$, Figure $3 D$ ) in the nontumor tissue samples. 
Table 1 Correlation of clinicopathologic characteristics with CD74 and MIF in HCC

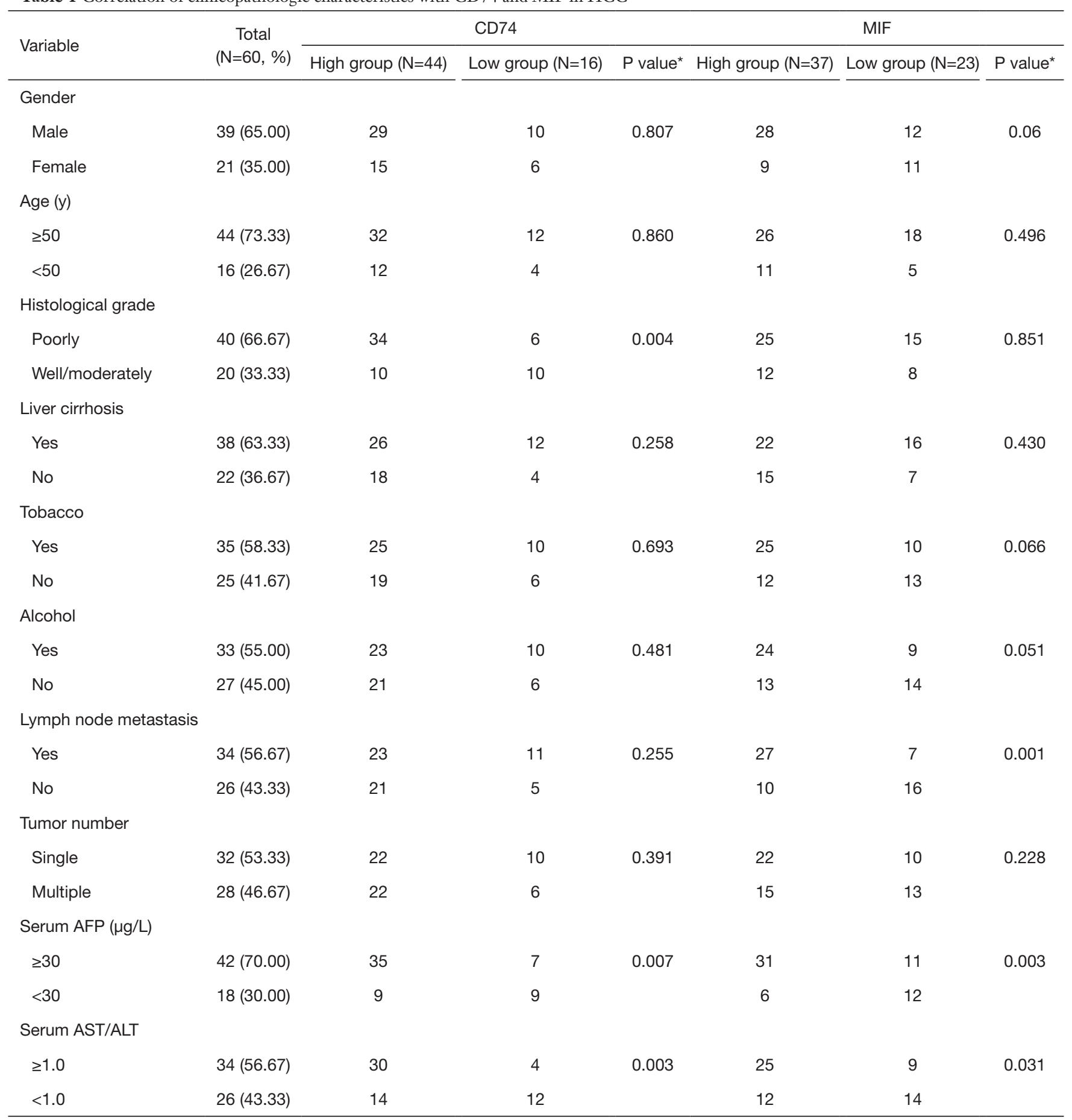

*, statistical significance determined by Pearson's Chi-squared test; high group, the CD74 or MIF ratio $\geq 1.0$; low group, the CD74 or MIF ratio <1.0. HCC, hepatocellular carcinoma; MIF, migration inhibitory factor; AFP, $\alpha$-fetoprotein; AST, aspartate aminotransferase; ALT, alanine aminotransferase. 

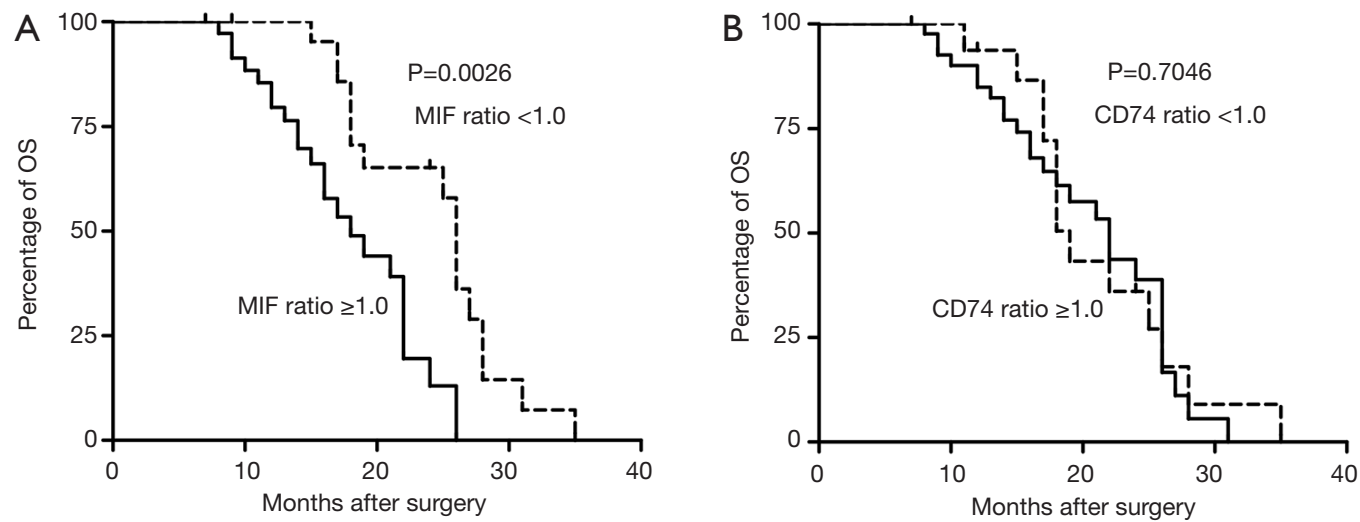

Figure 2 The level of CD74 and MIF correlates with overall HCC survival. (A) The high level of MIF (ratio $\geq 1.0$ ) was significantly correlated with the poor survival rate after surgery in patients; (B) the high level of CD74 (ratio $\geq 1.0$ ) was not significantly correlated with the poor overall survival rate after surgery in patients. The overall survival was done using Kaplan-Meier analysis. MIF, migration inhibitory factor; HCC, hepatocellular carcinoma.
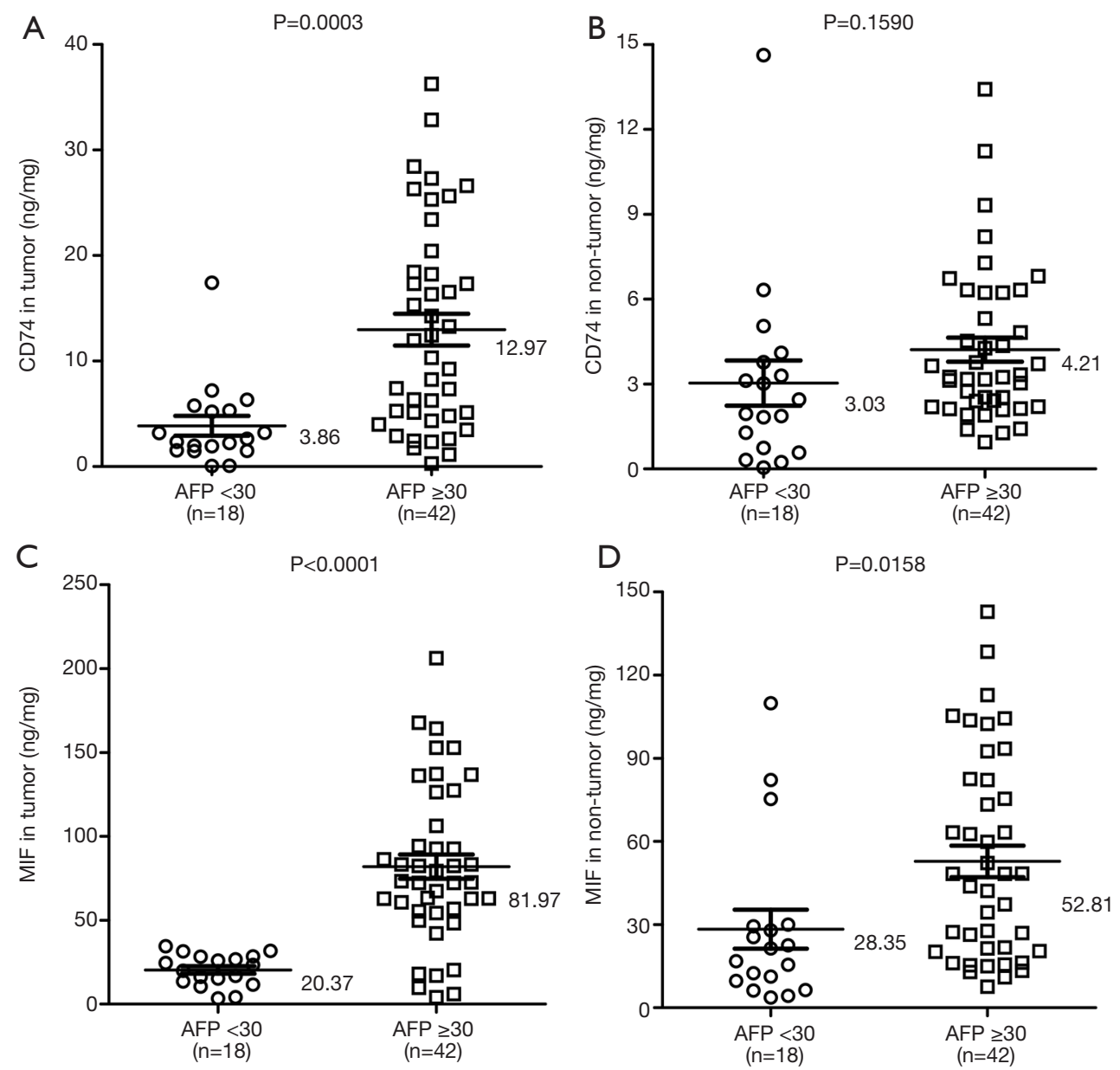

Figure 3 The level of CD74 and MIF correlated with serum AFP. (A) The level of CD74 was associated with serum AFP in tumor tissues; (B) the level of CD74 was not associated with serum AFP in non-tumor tissues; (C) MIF was associated with serum AFP in tumor tissues; (D) MIF was associated with serum AFP in non-tumor tissues. Statistical analysis was done using the unpaired $t$-test. MIF, migration inhibitory factor; AFP, $\alpha$-fetoprotein. 

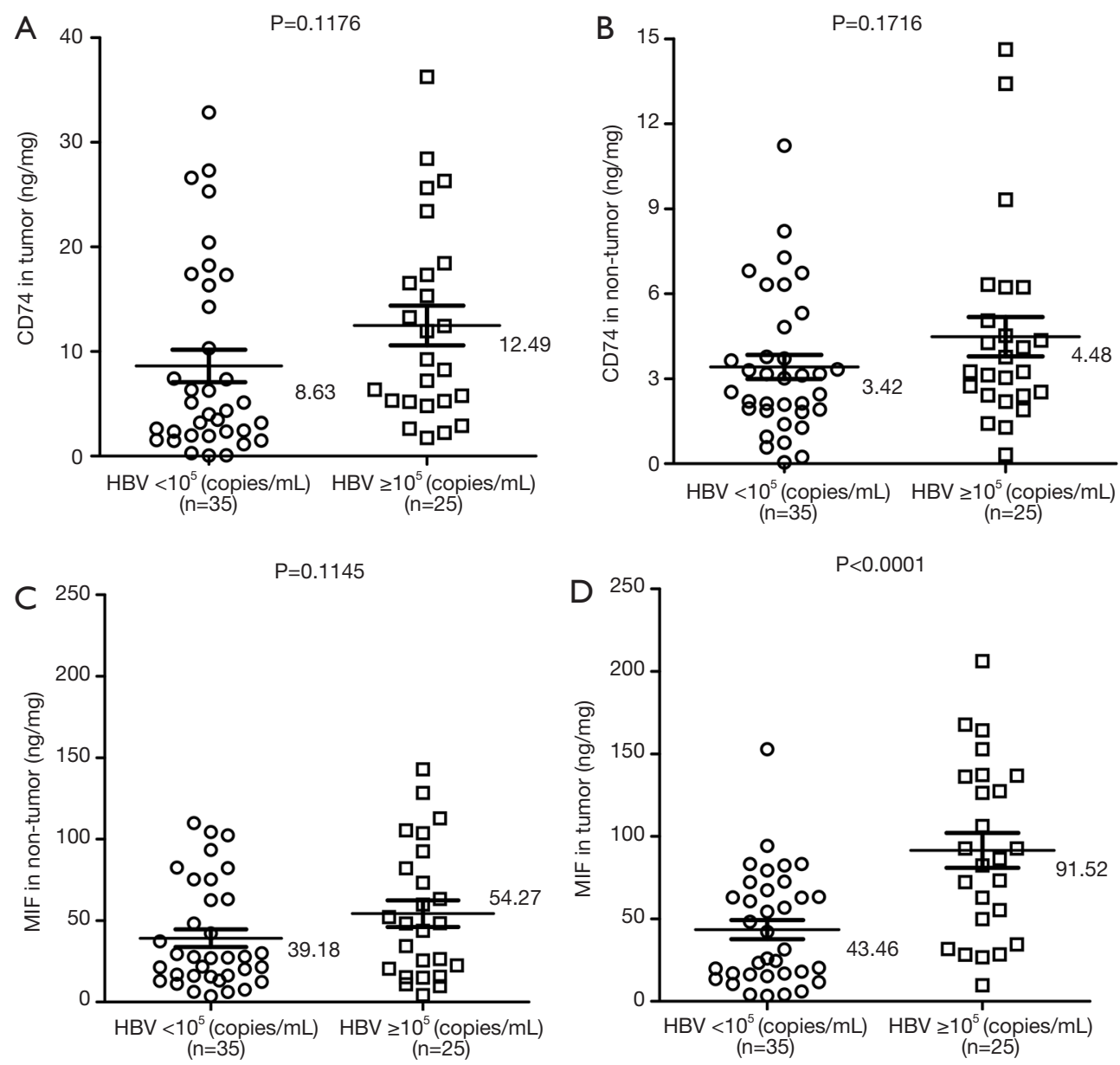

Figure 4 The level of CD74 and MIF associated with serum HBV DNA load. (A,B) The level of CD74 was not associated with high serum HBV DNA load in tumor tissues and in non-tumor tissues; (C,D) MIF was associated with high serum HBV DNA load in tumor tissues, but not in non-tumor tissues. Statistical analysis was done using the unpaired $t$-test. HBV, hepatitis B virus; MIF, migration inhibitory factor.

\section{The level of CD74 and MIF protein with serum HBV in $\mathrm{HBV}$-related $\mathrm{HCC}$}

Hepatitis B is an important factor for chronic hepatitis, cirrhosis and HCC in Asia. HBV replication is associated with the severity of disease and cirrhosis. We observed 25 cases $(41.67 \%)$ with a high HBV level $\left(\geq 10^{5}\right.$ copies $\left./ \mathrm{mL}\right)$ and 35 cases $(58.33 \%)$ with a low HBV level $\left(<10^{5}\right.$ copies $\left./ \mathrm{mL}\right)$. The results indicated that the mean concentration of CD74 demonstrated no significant difference between the HBV-high cases and HBV-low cases, in both tumor tissues or non-tumor tissues (Figure $4 A, B$ ). Furthermore, there was also no significant difference in both groups for MIF protein expression in non-tumor tissues (Figure 4C). However, HBV-related HCC cases with HBV-high level showed a higher level of MIF protein than the HBV-low group in tumor tissues $(91.52,95 \% \mathrm{CI}, 69.68-113.40$ vs. 43.46, 95\% CI, 31.75-55.17, $\mathrm{P}<0.0001$, Figure 4D). These results suggested that the elevated expression of MIF protein was associated with $\mathrm{HBV}$ replication in liver cancer.

\section{Elevated MIF is associated with high CD74 in $\mathrm{HBV}$-related $\mathrm{HCC}$}

To determine whether MIF is associated with CD74 protein expression, we defined the level of protein group as following: CD74-high, CD74-low; MIF-high, MIFlow. We found there was not a significant difference between MIF-high and MIF-low group cases for CD74 protein expression, in both tumor tissues or non-tumor tissues $(\mathrm{P}=0.1357, \mathrm{P}=0.1244$; Figure $5 A, B)$. However, for 

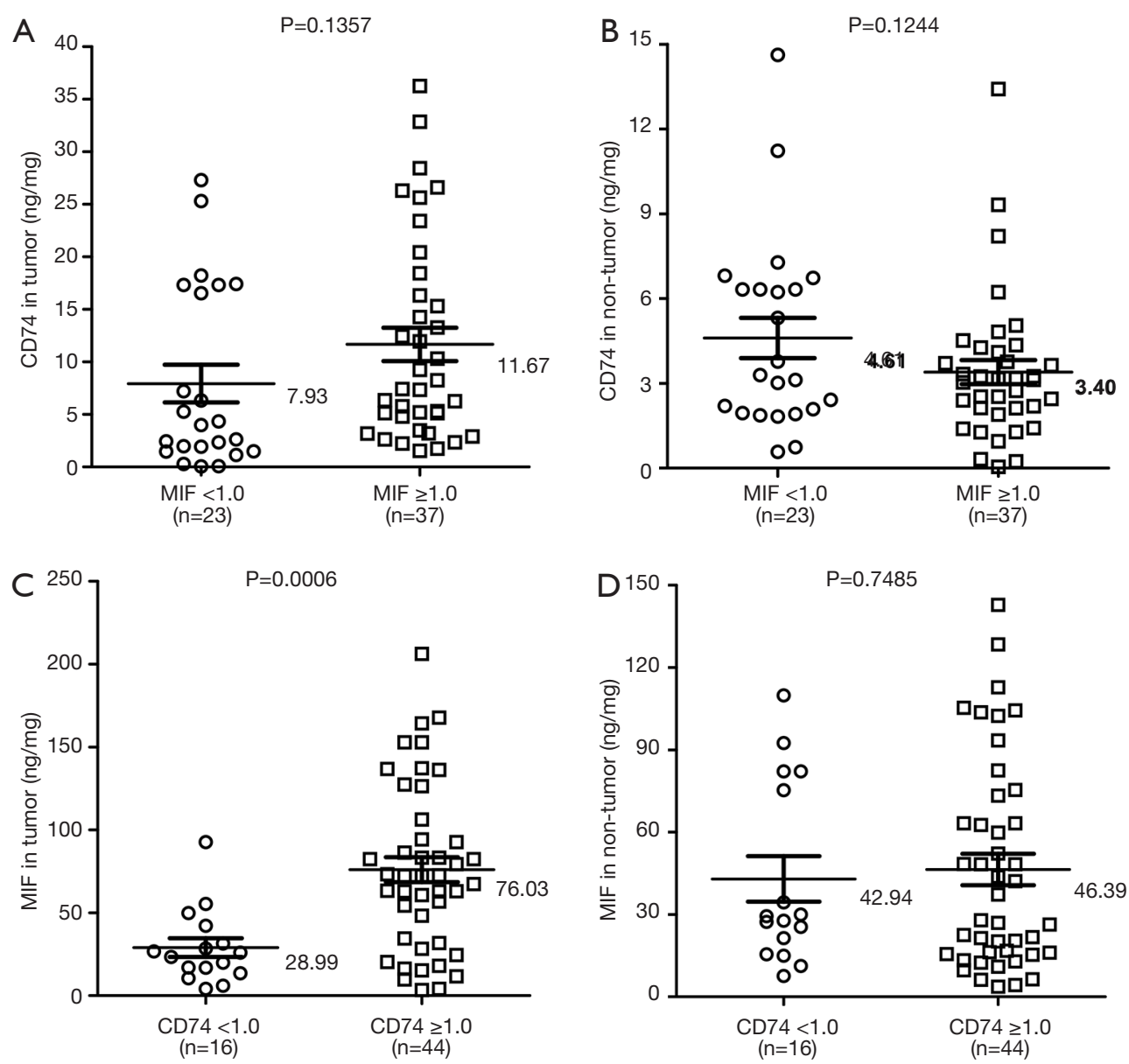

Figure 5 The association between high level of CD74 and high level of MIF in patients. (A,B) There was no significant difference between MIF-high and MIF-low group for CD74 expression in tumor tissues and in non-tumor tissues; (C,D) the MIF expression was significantly different between CD74-high and CD74-low group in tumor tissues, but not in non-tumor tissues. Statistical analysis was done using the unpaired t-test. MIF, migration inhibitory factor.

the level of MIF proteins, there was a significant difference between CD74-high and CD74-low group cases in tumor tissues $(\mathrm{P}=0.0006, \mathrm{P}=0.7485$; Figure $5 C, D)$. The mean level of MIF in CD74-high group was $76.03 \mathrm{ng} / \mathrm{mg}$, and $28.99 \mathrm{ng} / \mathrm{mg}$ in CD74-low group in tumor tissues.

For next step, we evaluated the levels of CD74 and MIF factor in all samples. There was a close correlation between CD74 and MIF levels in tumor tissues, in non-tumor tissues, and in all tissues $\left(\mathrm{R}^{2}=0.422, \mathrm{P}<0.0001\right.$, Figure 6 A; $\mathrm{R}^{2}$ $=0.412, \mathrm{P}<0.0001$, Figure $6 B ; \mathrm{R}^{2}=0.397, \mathrm{P}<0.0001$, Figure $6 C$; respectively). Our data indicated that the elevated levels of the MIF protein level were associated with the elevated levels of CD74 protein in HBV-associated HCC progression.

\section{Discussion}

The HBV has been considered an important etiological factor during the development of HCC (18). Multiple abnormal mechanisms can promote tumor progression due to an $\mathrm{HBV}$ infection, including gene expression and the cellular signaling processes (19). Chronic HBV infection results in a chronic inflammation of liver tissues and hepatocytes. Subsequently, inflammatory cells are recruited and activated, inducing the continued expression of cytokines to affect an inflammatory injury. Inflammation triggered by $\mathrm{HBV}$ is an important contributor to the promotion of fibrosis, cirrhosis, and HCC (20). The pro-inflammatory cytokines are released in response to an infection and inflammation via pattern recognition receptors, which are involved in the classical inflammatory 

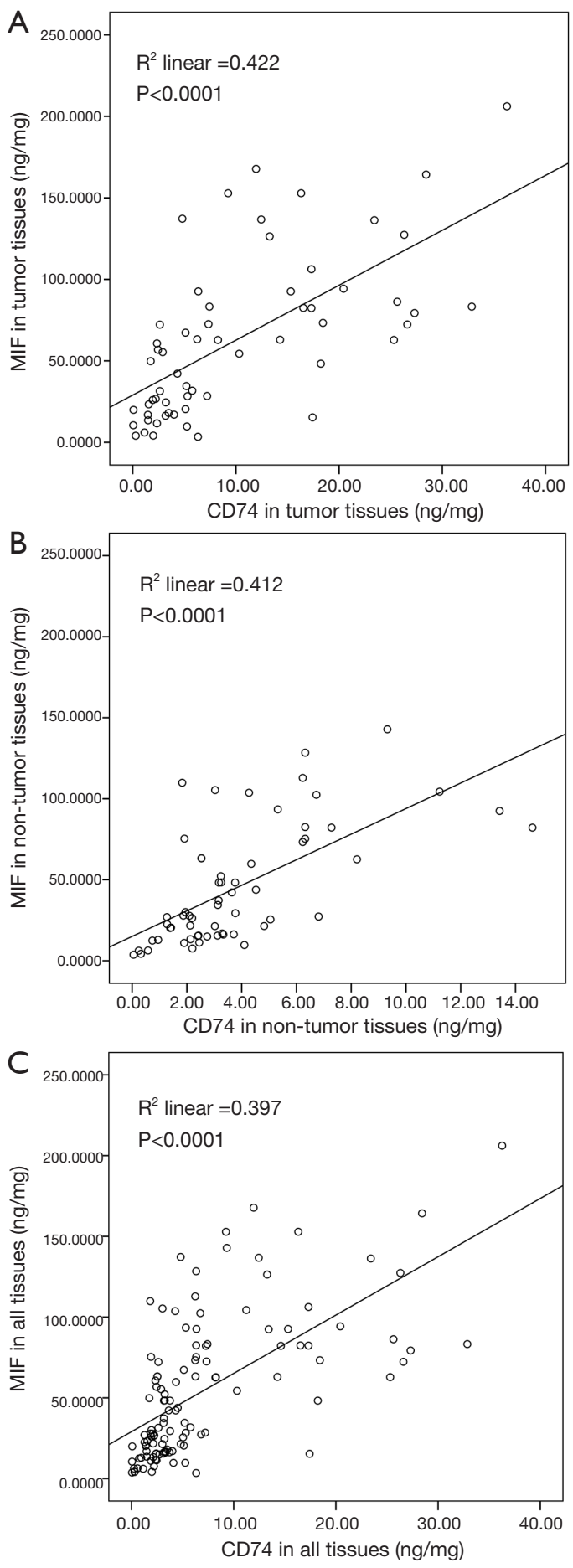

Figure 6 Correlation between CD74 level status and MIF expression. (A) Correlation between CD74 and MIF levels in tumor tissues; (B) correlation between CD74 and MIF level in non-tumor tissues; (C) correlation between CD74 and MIF level in all tissues. Statistical analysis was performed with linear regression. MIF, migration inhibitory factor.

pathway activation to modulate the tumor microenvironment. The tumor microenvironment is an indispensable element in the neoplastic process of inflammatory cells and the respective inducement of cytokine production (21).

A previous study indicated that elevated expression of MIF and CD74 could promote cell proliferation and it was found in the advanced gastric cancer by immunohistochemistry (22). Many studies have provided evidence showing that the hepatitis virus plays a role in liver tumorigenesis by provoking an inflammatory-mediated liver injury. In this study, we discussed the role of inflammation cytokines (CD74 and MIF) in HBV-related HCC development. We found an elevated level of CD74 and MIF protein in the tumor tissues versus the non-tumor tissues.

AFP is as one of serum biomarkers associated with liver cell proliferation related to the pathogenesis of HCC. The elevated ALT and AST levels are used as indicators of liver cell injury in liver cirrhosis, and also as serum markers for acute and chronic hepatocytes cytotoxicity $(23,24)$. Preoperative serum AST/ALT ratios and AFP were independently associated with an early recurrence of HCC after a curative resection (25). AST/ALT ratio has also been suggested to act as a fibrosis marker to reflect progressive liver functional impairment (26-28). Our results found that increased CD74 protein expression was associated with serum AFP level and AST/ALT ratio in HBV-related HCC patients. The high level of MIF protein was associated with lymph node metastasis, serum AFP level and AST/ ALT ratio. The results indicated that the high expression of CD74 protein was found in the AFP-high patients compared with the AFP-low group in tumor tissues. Yet, MIF expression was increased in the AFP-high patients for both tumor or non-tumor tissues. Only the high-level of MIF protein was associated with short-term survival months, but CD74 was lacking in its prognostic significance in HBV-related HCC cases. These results imply a clinical and prognostic relevance for the elevated levels of the MIF protein in HCC, which reflect the status of liver cell injury, inflammation, or proliferation in the development of HCC.

The serum HBV DNA level is an important HCC predictor. The serum HBV DNA $\geq 10^{5}$ copies/mL has been proposed as having an active chronic HBV state by the NIH (29). High HBV DNA viral load have also been proposed as a factor for high-risk chronic hepatitis B patients (30). Our results found that there was no significant difference between HBV-high cases and $\mathrm{HBV}$-low cases for $\mathrm{CD} 74$ protein in the tissue samples. But, a higher level of the MIF protein was found in the HBV-high level (HBV DNA $\geq 10^{5}$ copies $/ \mathrm{mL}$ ) group in their tumor tissue 
samples, which suggested that MIF protein could by induced by high $\mathrm{HBV}$ replication in liver cancer. However, these results implied that MIF and CD74 could act as a predictor related to impaired liver function and the host immunoreactivity to HBV.

The imbalance of pro-inflammatory and anti-inflammatory cytokine has been associated with the rate of metastasis and a poor clinical significance in the microenvironment of HCC patients (3). The ratio of serum CD74/MIF has been correlated with serum ALT in relapse patients with autoimmune liver diseases (31). There was a close correlation between the CD74 and MIF levels in both tumor and non-tumor tissues. The level of MIF protein in patients with elevated CD74 expression was significantly higher than CD74-low group cases in tumor tissues. Our data indicated that the elevated level of MIF protein level was associated with the elevated level of $\mathrm{CD} 74$ protein in $\mathrm{HBV}$-associated HCC progression.

Increased CD74 and MIF protein levels have also been found in the patients with autoimmune hepatitis and primary biliary cirrhosis versus the healthy controls by ELISA. In one study, CD74 was released in hepatic stellate cells and targeted MIF signal transduction activity, which may play a role in the pathogenesis of autoimmune liver disease (31). Our data provided a novel insight into $\mathrm{HBV}$-mediated liver pathogenesis by the CD74-MIF pathway in HCC. The quantification of serum markers and pro-inflammatory cytokines may be helpful for clinical patients. However, various inflammation cytokines are changing dynamically during the hepatopathogenic progression. It is helpful to provide information related to liver fibrosis and cirrhosis to patients based on the MIF and CD74 measurements in the early stage of HCC. The MIF and CD74 protein may be risk predictors for immune tolerance and immune clearance in the tumor microenvironment promoting $\mathrm{HCC}$ carcinogenesis and progression.

In sum, HBV may influence MIF expression by pro-inflammatory cytokines induced in a tumor microenvironment. Our data indicated that $\mathrm{HBV}$ might have increased MIF protein levels. These data points suggest a potential role of the CD74-MIF pathway in HBVassociated HCC. The data may also elucidate how MIF can contribute to hepatocarcinogenesis as an abundant inflammation cytokine through inducement by the HBV virus. However, further studies are needed to fully explore exactly how pro-inflammatory cytokines are involved in the multiple pathways of $\mathrm{HBV}$-mediated liver pathogenesis.

\section{Acknowledgments}

Funding: This study was supported by the Natural
Science Foundation of Jiangsu (Grant No.BE2016656), the Changzhou Sci \& Tech Program, China (Grant No.CJ20179029 and CE20175037), the Health Talents Project for Jiangsu, China (Grant No. ZDRCC2016020), the High-level Health Talents of Changzhou City (Grant No. 2016CZLJ009), and Changzhou Municipal Commission of Health and Family Planning (ZD201816).

\section{Footnote}

Conflicts of Interest: TAll authors have completed the ICMJE uniform disclosure form (available at http://dx.doi. org/10.21037/tcr.2018.11.21). The authors have no conflicts of interest to declare.

Ethical Statement: The authors are accountable for all aspects of the work in ensuring that questions related to the accuracy or integrity of any part of the work are appropriately investigated and resolved. The study was conducted in accordance with the Declaration of Helsinki (as revised in 2013). All of patients gave written informed consent, and approval was given by the Ethics Committee of Changzhou Cancer Hospital of Soochow University (No. 2016006).

Open Access Statement: This is an Open Access article distributed in accordance with the Creative Commons Attribution-NonCommercial-NoDerivs 4.0 International License (CC BY-NC-ND 4.0), which permits the noncommercial replication and distribution of the article with the strict proviso that no changes or edits are made and the original work is properly cited (including links to both the formal publication through the relevant DOI and the license). See: https://creativecommons.org/licenses/by-nc-nd/4.0/.

\section{References}

1. Xie Y. Hepatitis B Virus-Associated Hepatocellular Carcinoma. Adv Exp Med Biol 2017;1018:11-21.

2. Szabo G, Lippai D. Molecular hepatic carcinogenesis: impact of inflammation. Dig Dis 2012;30:243-8.

3. Budhu A, Wang XW. The role of cytokines in hepatocellular carcinoma. J Leukoc Biol 2006;80:1197-213.

4. Bucala R, Shachar I. The integral role of CD74 in antigen presentation, MIF signal transduction, and $\mathrm{B}$ cell survival and homeostasis. Mini Rev Med Chem 2014;14:1132-8.

5. Conroy H, Mawhinney L, Donnelly SC. Inflammation and cancer: macrophage migration inhibitory factor (MIF)--the potential missing link. QJM 2010;103:831-6. 
6. Javeed A, Zhao Y, Zhao Y. Macrophage-migration inhibitory factor: role in inflammatory diseases and graft rejection. Inflamm Res 2008;57:45-50.

7. Choi JW, Kim Y, Lee JH, et al. CD74 expression is increased in high-grade, invasive urothelial carcinoma of the bladder. Int J Urol 2013;20:251-5.

8. Simpson KD, Templeton DJ, Cross JV. Macrophage migration inhibitory factor promotes tumor growth and metastasis by inducing myeloid-derived suppressor cells in the tumor microenvironment. J Immunol 2012;189:5533-40.

9. Lechien JR, Nassri A, Kindt N, et al. Role of macrophage migration inhibitory factor in head and neck cancer and novel therapeutic targets: A systematic review. Head Neck 2017;39:2573-84.

10. Liu RM, Sun DN, Jiao YL, et al. Macrophage migration inhibitory factor promotes tumor aggressiveness of esophageal squamous cell carcinoma via activation of Akt and inactivation of GSK3beta. Cancer Lett 2018;412:289-96.

11. Zhang L, Ye SB, Ma G, et al. The expressions of MIF and CXCR4 protein in tumor microenvironment are adverse prognostic factors in patients with esophageal squamous cell carcinoma. J Transl Med 2013;11:60.

12. Gil-Yarom N, Radomir L, Sever L, et al. CD74 is a novel transcription regulator. Proc Natl Acad Sci U S A 2017; 114:562-7.

13. Leng L, Metz CN, Fang Y, et al. MIF signal transduction initiated by binding to CD74. J Exp Med 2003;197:1467-76.

14. Grieb G, Kim BS, Simons D, et al. MIF and CD74 suitability as clinical biomarkers. Mini Rev Med Chem 2014;14:1125-31.

15. Otterstrom C, Soltermann A, Opitz I, et al. CD74: a new prognostic factor for patients with malignant pleural mesothelioma. Br J Cancer 2014;110:2040-6.

16. Richard V, Kindt N, Saussez S. Macrophage migration inhibitory factor involvement in breast cancer (Review). Int J Oncol 2015;47:1627-33.

17. Ren Y, Tsui HT, Poon RT, et al. Macrophage migration inhibitory factor: roles in regulating tumor cell migration and expression of angiogenic factors in hepatocellular carcinoma. Int J Cancer 2003;107:22-9.

18. Arzumanyan A, Reis HM, Feitelson MA. Pathogenic mechanisms in HBV- and HCV-associated hepatocellular carcinoma. Nat Rev Cancer 2013;13:123-35.

19. Levrero M, Zucman-Rossi J. Mechanisms of HBV-induced hepatocellular carcinoma. J Hepatol 2016;64:S84-101.

20. Wang M, Xi D, Ning Q. Virus-induced hepatocellular carcinoma with special emphasis on HBV. Hepatol Int 2017;11:171-80.

21. Wang X, Hassan W, Jabeen Q, et al. Interdependent and independent multidimensional role of tumor microenvironment on hepatocellular carcinoma. Cytokine 2018;103:150-9.

22. Zheng YX, Yang M, Rong TT, et al. CD74 and macrophage migration inhibitory factor as therapeutic targets in gastric cancer. World J Gastroenterol 2012;18:2253-61.

23. Dufour DR, Lott JA, Nolte FS, et al. Diagnosis and monitoring of hepatic injury. I. Performance characteristics of laboratory tests. Clin Chem 2000;46:2027-49.

24. Sheth SG, Flamm SL, Gordon FD, et al. AST/ALT ratio predicts cirrhosis in patients with chronic hepatitis $\mathrm{C}$ virus infection. Am J Gastroenterol 1998;93:44-8.

25. Wang ZX, Jiang CP, Cao Y, et al. Preoperative serum liver enzyme markers for predicting early recurrence after curative resection of hepatocellular carcinoma. Hepatobiliary Pancreat Dis Int 2015;14:178-85.

26. Lin YJ, Lee MH, Yang HI, et al. Predictability of liverrelated seromarkers for the risk of hepatocellular carcinoma in chronic hepatitis B patients. PLoS One 2013;8:e61448.

27. Giannini E, Risso D, Botta F, et al. Validity and clinical utility of the aspartate aminotransferase-alanine aminotransferase ratio in assessing disease severity and prognosis in patients with hepatitis $\mathrm{C}$ virus-related chronic liver disease. Arch Intern Med 2003;163:218-24.

28. Lee MH, Yang HI, Liu J, et al. Prediction models of longterm cirrhosis and hepatocellular carcinoma risk in chronic hepatitis B patients: risk scores integrating host and virus profiles. Hepatology 2013;58:546-54.

29. Lok AS, McMahon BJ. Chronic hepatitis B. Hepatology 2007;45:507-39.

30. Lok AS, Heathcote EJ, Hoofnagle JH. Management of hepatitis B: 2000--summary of a workshop. Gastroenterology 2001;120:1828-53.

31. Assis DN, Leng L, Du X, et al. The role of macrophage migration inhibitory factor in autoimmune liver disease. Hepatology 2014;59:580-91.

Cite this article as: $\mathrm{Lu} \mathrm{M}, \mathrm{Xu}$ Y, He G, Liu Q, Zhu J, Zhang C. The expression of $\mathrm{CD} 74$ and macrophage migration inhibitory factor protein is upregulated in hepatitis B virus-related hepatocellular carcinoma. Transl Cancer Res 2018;7(6):15371547. doi: $10.21037 /$ tcr.2018.11.21 